\title{
Erratum to: Imaging tumor angiogenesis in breast cancer experimental lung metastasis with positron emission tomography, near-infrared fluorescence, and bioluminescence
}

\author{
Yin Zhang • Hao Hong • Tapas R. Nayak • Hector F. Valdovinos • \\ Duane V. Myklejord · Charles P. Theuer · Todd E. Barnhart • \\ Weibo Cai
}

Published online: 16 May 2013

(C) Springer Science+Business Media Dordrecht 2013

Erratum to: Angiogenesis

DOI 10.1007/s10456-013-9344-y

Unfortunately, in the original publication of the article, histology images of lung and spleen were incorrectly used in Fig. 6. The correct version of the Fig. 6 is provided in this Erratum.

The online version of the original article can be found under doi:10.1007/s10456-013-9344-y.

Y. Zhang · H. F. Valdovinos · T. E. Barnhart · W. Cai ( $\square)$ Department of Medical Physics, University of

Wisconsin - Madison, Room 7137, 1111 Highland Avenue, Madison, WI 53705, USA

e-mail: wcai@uwhealth.org

H. Hong · T. R. Nayak · D. V. Myklejord · W. Cai Department of Radiology, University of Wisconsin - Madison, Room 7137, 1111 Highland Avenue, Madison, WI 53705, USA

C. P. Theuer

TRACON Pharmaceuticals, Inc., San Diego, CA, USA

W. Cai

University of Wisconsin Carbone Cancer Center, Madison, WI,

USA 
Fig. 6 Immunofluorescence staining of the fLuc-4T1 tumor lung, normal mouse lung, liver, and spleen tissue sections.

Green CD105; red CD31; blue DAPI. All images were acquired under the same conditions and displayed at the same scale. Magnification: $\times 200$. Scale bar $50 \mu \mathrm{m}$
4T1 lung
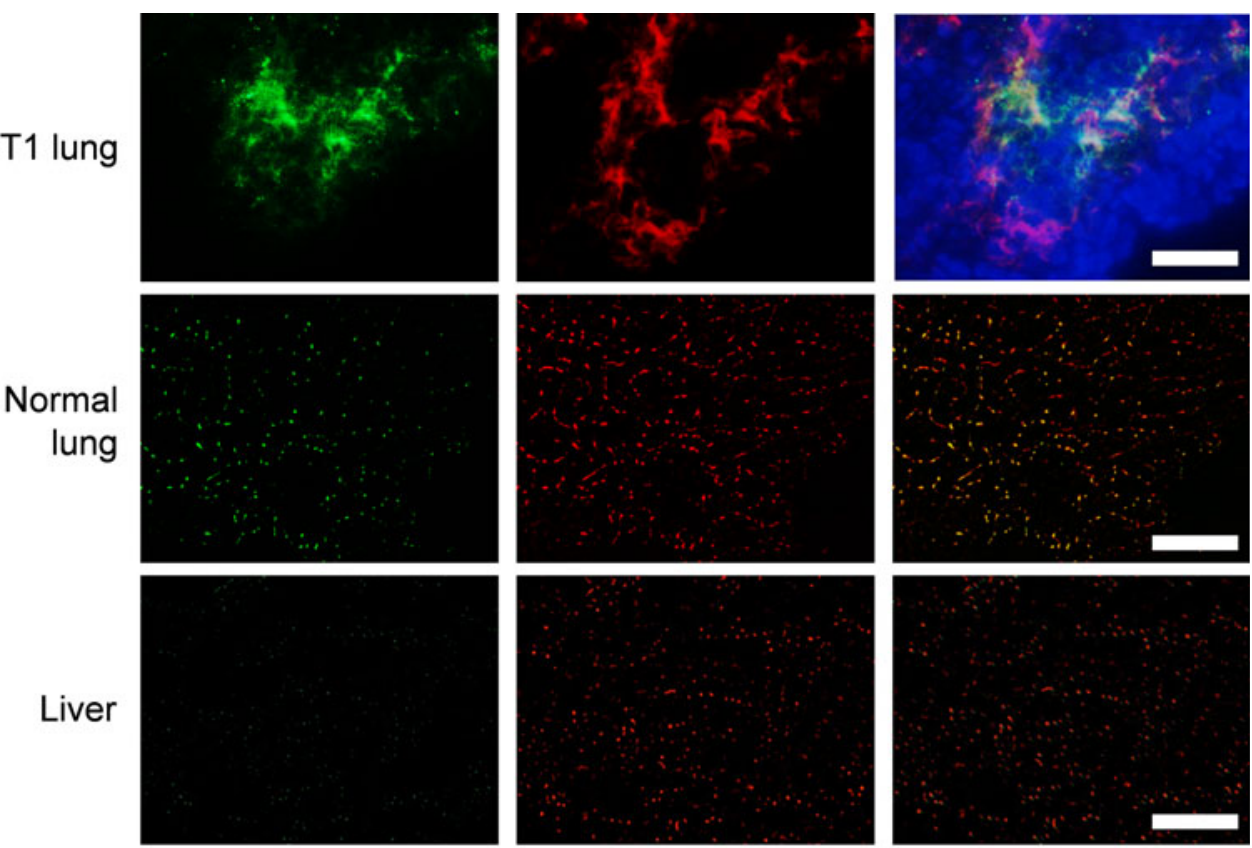

Liver
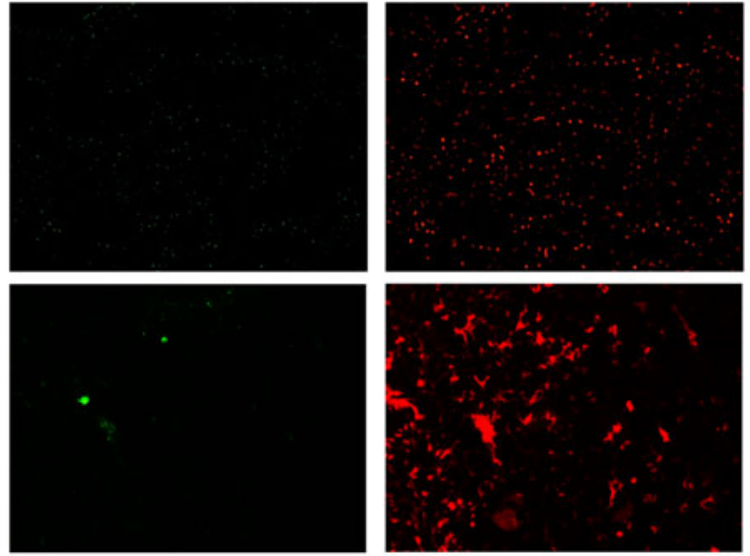

CD31

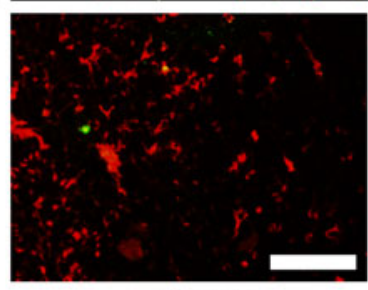

Merge 